\title{
Banded Texture Induced by an Electric Field in Polymeric Liquid Crystalline Solution
}

\author{
Kazuhiro Monzen, Kazuyuki Hiraoka, Yoshiko Uematsu, ${ }^{\dagger}$ \\ and Munehiro DATE* \\ Tokyo Institute of Polytechnics, 1583 Iiyama, Atsugi-shi, Kanagawa 243-0297, Japan \\ * The Institute of Physical and Chemical Research, \\ 2-1 Hirosawa, Wako-shi, Saitama 351-0198, Japan
}

(Received November 26, 1997)

\begin{abstract}
Using polarizing microscopy, we investigated the textural transformation and development of banded texture for cholesteric (nematic) liquid-crystalline solutions of poly( $\gamma$-benzyl glutamate) (PBG)s in an in-plane electric field. The threshold value for producing the uniform director orientation depends on cell thickness, $d$, and is inversely proportional to $d^{1 / 2}$. In the solution aligned uniaxially by a de field, the transient banded texture appears perpendicular to the field soon after inversion of the field direction. By using video-recording technique, detailed analysis of the texture can be performed and the following results are obtained: the appearance of banded texture is related to rotation of the director during realignment and two peaks of the optical intensity transmitted through the sample between crossed polarizers appear until the reorientation is completed; the band spacing remains constant during realignment and depends on the applied field strength; being a decreasing function of field strength. The effect of cholesteric twist on the kinetics of director rotation is studied for solutions of poly $(\gamma$-benzyl-L-glutamate) (PBLG), poly( $\gamma$-benzyl-D-glutamate) (PBDG) and racemic mixtures. A cholesteric twist was noted to affect kinetics of director rotation.

KEY WORDS Poly $(\gamma$-benzyl-L-glutamate) / Polymeric Liquid Crystalline / Electric Field / Banded Texture / Threshold Value /
\end{abstract}

One distinctive phenomenon in the flow of lyotropic liquid crystalline polymers in the nematic or the cholesteric state is negative steady-state first normal stressdifference in shearing flow over a range of shear rates. For an isotropic fluid, the first normal stress difference, $N_{1}$, is zero or positive over all rates of shear. However, Kiss and Porter found that in poly $(\gamma$-benzyl-Lglutamate) (PBLG) solutions, positive values of $N_{1}$ were seen at low and high shear rates and negative values at intermediate shear rates. ${ }^{1}$ They also found that the banded texture appeared at intermediate shear rates. The appearance of banded texture, as a striped pattern, perpendicular to the flow direction is particularly interesting. The characteristic banded texture has been observed during or after shear, in various main chain liquid-crystalline polymers, ${ }^{2-34}$ thermotropic systems as well as lyotropics. ${ }^{10,35-40}$ Many models have been proposed to explain the mechanism of band formation. ${ }^{23,41,42}$ However, the mechanism is not yet completely understood. Most theories consider that such behavior in lyotropic polymer liquid-crystals results from coupling between molecular tumbling motion and local molecular rotation during shear. ${ }^{43-46}$ For band formation, Patlazhan et al. represented recently a model of defects at low shear rate, grown with increasing shear, on the basis of the results of depolarized light scattering observed at low shear rates for lyotropic and thermotropic polymer liquid-crystals. ${ }^{47}$ The model consists of a twist loop, fluctuating around the shearing plane, with a director varying from the flow direction to vorticity axis. Therefore, the striped pattern is transient, unless an extremely low shear field is used.

To minimize the molecular displacement with flow and to obtain quantitative, time independent, information of

\footnotetext{
$\dagger^{\dagger}$ To whom correspondence should be addressed.
}

banded texture, we used an electric field instead of a shear field. The threshold values for band formation and band spacing is much more reproducible by replacing a shear field with an electric field.

This paper provides a quantitative description of domain size induced by electric fields instead of a shear field and the qualitative interpretation for the rotational motion of directors in a striped domain.

\section{EXPERIMENTAL}

\section{Materials}

All samples of PBLG and poly( $\gamma$-benzyl-D-glutamate) (PBDG) used in this experiment were prepared by polymerization of $N$-carboxy- $\gamma$-benzyl glutamate anhydride (NCA). ${ }^{48}$ The molecular weights of PBLG and PBDG estimated from viscosity measurement are as follows: PBLG, 48000 and 96000; PBDG, 106000. For all solutions of poly $(\gamma$-benzyl glutamate) (PBG) in 1,1,2-trichloroethane (TCE), the concentration amounted to $0.18 \mathrm{v} / \mathrm{v}$, in order to afford anisotropic solutions.

\section{Experimental Methods}

The solution, after incubation at room temperature at least for $24 \mathrm{~h}$, was sandwiched between two parallel glass plates coated partially with $\mathrm{Au}$ as electrodes, as shown in Figure 1a. The level dimensions of cell were $0.5 \mathrm{~cm} \times 0.5 \mathrm{~cm}$ and cell thickness was controlled by inserting Teflon spacers between glass plates, at 50 to $500 \mu \mathrm{m}$.

As shown in Figure $1 \mathrm{~b}$, the cell was mounted on the stage of a polarization microscope (OLYMPUS BH-2) between two polarizers. The transmission axis of the incident polarized light was parallel to the electric field direction and that of the analyzer was perpendicular to it. A video camera was mounted on top of the micro- 


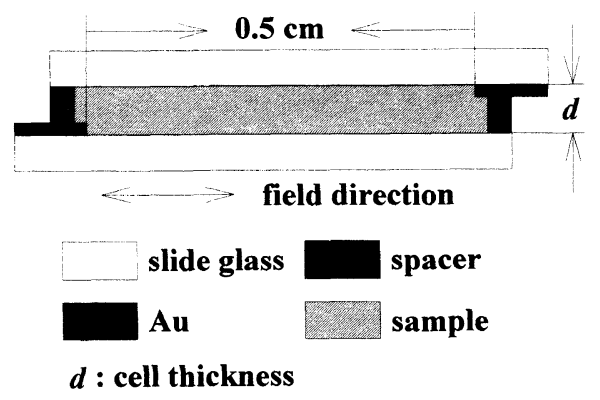

(a)

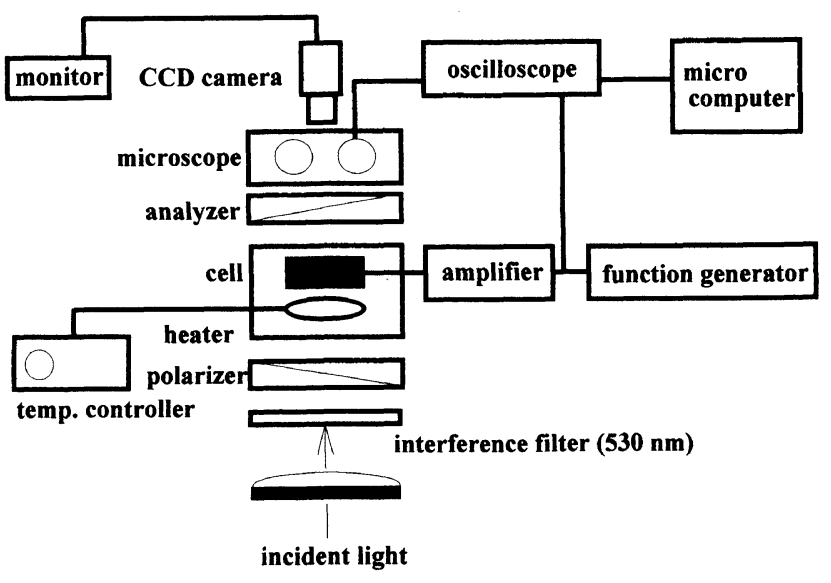

(b)

Figure 1. (a) Cell structure and (b) schematic representation of the experimental setup.

scope, permitting the video recording of a constantly varying texture. Individual frames were digitized at a fixed interval. The frame grabber has a resolution of $512 \times 480$ pixels with 256 gray scales. The rotational motion of the director in the domains was followed by the light intensity transmitted through a cell between crossed polarizers, using a photomultiplier attached to a microscope. The data of the transmitted light intensity was inputted into a micro computer (NEC $9801 \mathrm{VM}$ ) and analyzed.

\section{RESULTS AND DISCUSSION}

\section{Threshold Value}

The freshly prepared sample, sandwiched between two microscope slides separated by Teflon spacer, was left at room temperature for $24 \mathrm{~h}$ before applying an electric field. The samples were kept without any appreciable loss of solvent. The textures observed after leaving for $24 \mathrm{~h}$, depending on cell thickness, varied to a homeotropic nematic monodomain.

The threshold dc value for the textural transformation of each specimen with various thicknesses of cell for PBLG $\left(M_{w}=48000\right)$ liquid crystalline solution was measured, using a polarizing optical microscope, for each sample with stepping up dc field strength by $5 \mathrm{~V} \mathrm{~cm}^{-1}$ of slice every $30 \mathrm{~min}$. When electric field strength exceeded a certain value, $V_{1 \mathrm{c}}$, it was observed by the microscope that texture was gradually transformed, where the transmitted intensity through the crossed polarizers began to increase. When the applied field strength exceeded a value higher than $V_{1 \mathrm{C}}$, the transmitted in-

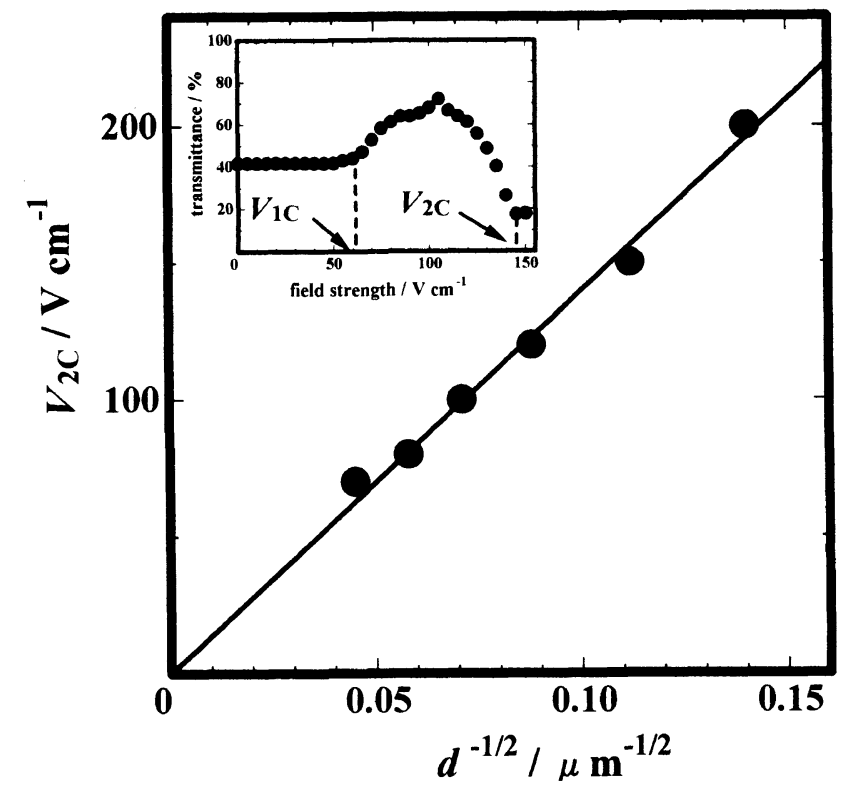

Figure 2. Variation of threshold, $V_{2 \mathrm{C}}$, with cell thickness, $d$, for PBLG $\left(M_{w}=48000\right)$ liquid crystalline solution. The variation of transmittance with applied field strength for $80 \mu \mathrm{m}$ of cell thickness is shown in the framed figure.

tensity decreased drastically and showed a minimum at the field strength, $V_{2 \mathrm{C}}$, where the field direction was parallel to one of the two crossed polarizers. An example of variation of transmittance in this process at $80 \mu \mathrm{m}$ of cell thickness is shown in Figure 2. The direction of preferred alignment induced by the external field, even though there was some short-range deviation, was recognized clearly and the director (birefringence axis) aligned roughly parallel to the field. The threshold, $V_{2 \mathrm{C}}$, was defined as the field strength at which the extinction of transmitted light occurred. The visual field became darkest and transmittance showed a minimum, under the experimental conditions. Generally, $V_{2 \mathrm{C}}$ lowered with increasing cell thickness, as shown in Figure 2. While the field strength for the initial instability of texture, $V_{1 \mathrm{c}}$, depended scarcely on the cell thickness at more than $200 \mu \mathrm{m}$ or less than $130 \mu \mathrm{m}, V_{1 \mathrm{c}}$ was inversely proportional to the square root of cell thickness, $d^{1 / 2}$, though the data points were not enough. The existence of defects and textural inhomogeneity in the former cell, at thickness more than $200 \mu \mathrm{m}$, may cause a decline in the apparent threshold for the initial instability. In the cell with the thickness equal to or less than $130 \mu \mathrm{m}$, there was exclusively the homeotropic texture with some or a few defects without any field. The decrease of cell thickness made the number of defects decrease. Homeotropic alignment should be stabilized by certain electrostatic interaction of the polar group at the molecular end of PBLG with ionic charges on the glass plate. When the sample thickness was $0.22 \mu \mathrm{m}$, excellent homeotropic alignment of PBLG molecules $\left(M_{w}=48000\right)$ was possible. For such samples, the critical fields, $V_{1 \mathrm{C}}$ and $V_{2 \mathrm{C}}$, were as high as 350 and $1160 \mathrm{~V} \mathrm{~cm}^{-1}$ respectively, even though the cell form was slightly different from that shown in Figure 1a. ${ }^{49}$ In Figure 2, the critical field strength, $V_{2 \mathrm{C}}$, is inversely proportional to $d^{1 / 2}$, as also for Parodi type transition apparently. ${ }^{50}$ Goscianski et al. found that the threshold varies as 


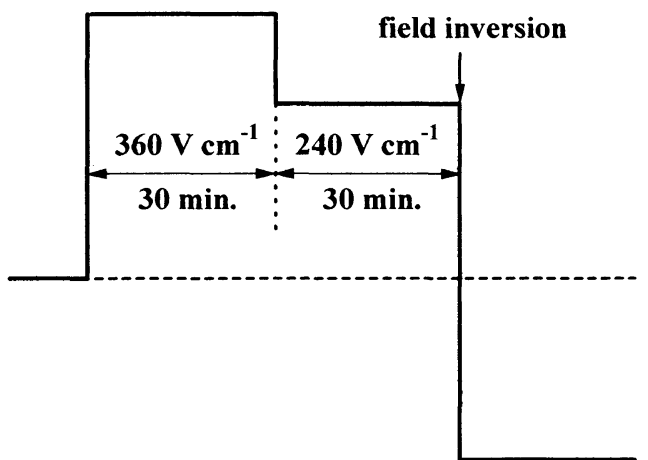

Figure 3. Example of the sequence of the applied field, where the desired value is $240 \mathrm{~V} \mathrm{~cm}^{-1}$.

$d^{-1 / 2}$ for transitions of molecular orientation induced by electric and magnetic field in the smectic A phase. ${ }^{51}$ They also found a regular striped pattern just above the threshold very close to $T_{\mathrm{SN}}$ and transition from the Freedericksz to Parodi type. The Parodi type transition in a nematic liquid crystal was also found by Allender et $a l .{ }^{52}$ and Frisken and Palffy-Muhoray. ${ }^{53}$ Using the sample homeotropically aligned between glass plates, an additional magnetic field was applied perpendicular to the plates and then an electric field was applied parallel to the plates. Frisken and Palffy-Muhoray observed the momentary appearance of the stripes parallel to the electrodes and perpendicular to the magnetic field, due to periodic modulation of director at the electric field-induced transition. ${ }^{54}$ In the experimental coordinate, the effects of magnetic field may be compared to surface stabilizing effects in our case. The relationship between the threshold values, $V_{1 \mathrm{C}}$ and $V_{2 \mathrm{C}}$, and the cell thickness, $d$, is apparently similar to that of Frisken and Palffy-Muhoray. However, the polydomain texture and imperfect alignment of an initial stage are involved in the transformational process in our system. It is considered that a quantitative relationship between the threshold values, $V_{1 \mathrm{C}}$ and $V_{2 \mathrm{C}}$, and $d$ is still highly fluid, depending on experimental conditions including the cell thickness.

\section{Banded Texture Observed by a Microscope}

The value higher than the critical field strength, $V_{2 \mathrm{C}}$, was applied to a sample beforehand. At first, the dc field strength of $360 \mathrm{~V} \mathrm{~cm}^{-1}$ was chosen as the initial field to be applied to samples for $30 \mathrm{~min}$ to provide roughly uniform orientation. The desired value was applied to the sample for $30 \mathrm{~min}$. Finally, the field direction was inverted, maintaining the same field strength. An example of applying field sequence is shown in Figure 3.

By polarization microscope, the appearance of the transient banded texture in a sample could be observed soon after the field direction was reversed. As examples of the typical banded texture appeared after an inversion of field, photomicrographs of PBDG $\left(M_{w}=106000\right)$ liquid crystalline solution after inverting a dc field strength of $40 \mathrm{~V} \mathrm{~cm}^{-1}$ are shown in Figure 4 , where cell thickness is $50 \mu \mathrm{m}$. The photomicrographs were taken under the condition which the wave plate was inserted diagonally between crossed polarizers and the lower polarizer was parallel to the field direction. Fine bands perpendicular to the field direction as shown in Figure 4a, appear soon after an inversion of field direction. With time, the bands faded as shown in Figure $4 \mathrm{~b}$ and were replaced by a uniform red field, i.e., dark field under the condition which the wave plate was not inserted, at once as shown in Figure $4 \mathrm{c}$ and then, after a while, they regenerated again with the same width and shape as shown in Figure 4a, except for the band colors observed with inserting a wave plate, i.e., the yellow bands in Figure $4 \mathrm{a}$ are replaced by blue ones in Figure $4 \mathrm{~d}$ and blue bands in the former by yellow ones in the latter. Soon, the bands faded again as shown in Figure 4e. The director in bands is flipped out of the field direction and it rotates clockwise and counterclockwise in the adjacent bands respectively.

The banded texture appeared even at the values less than the critical field strength, $V_{2 \mathrm{C}}$, that is to say, reorientation of the directors could be taken even at lower values than the critical field strength. This implies that an electrostatic interaction between polar ends of polymer and ionic groups of glass surface affects strongly the critical field strength for a virgin sample but it becomes less effective on rotational motion of director after the textural transformation has been induced by an electric field.

Our visual observations of band formation after an inversion of $\mathrm{dc}$ electric field are qualitatively similar to the observations of early works in a shear field, under which the transient band was observed after cessation of steady shear, but we could obtain more quantitative results under application of an electric field. In the early works with shear field, it was noticed that the development of banded textures in polymeric liquid crystalline (PLC) solutions depended on several molecular parameters and processing conditions; molecular weight of polymer, type of solvent, concentration, sample thickness, shear rates, duration of shear and method of applying shear. ${ }^{14,18,55-58}$ To avoid these effects and to provide the quantitative description of banded texture, molecular parameters and processing conditions are held in the limited values and conditions in the present work $^{59}$ as possible.

At the beginning, we focused our interest on the characteristic band width or the domain size, $L$, in unit of length. The length, $L$, can be thought as a correlation length. That is to say, in a band, it is considered that there is a roughly uniform region concerning orientation. The linear relationship between shear stress, $F$, and $L^{-2}$ in the shear field ${ }^{46}$ was considered that $L^{-2}$ was the total length of the disclination line per unit volume of the sample since the disclination lines permeated over the sample. The relationship between the applied field strength, $V$, and band size, $L$, for PBLG $\left(M_{w}=48000\right)$ sample at $80 \mu \mathrm{m}$ of cell thickness is shown in Figure 5. In the relatively low field strength region less than $240 \mathrm{~V} \mathrm{~cm}^{-1}, V$ is inversely proportional to $L^{2}$. However, in the higher field strength region, the band size depends strongly on the field strength and $L \propto V^{-2}$, departing from scaling law.

\section{Kinetics of Director Rotation}

The banded texture could be observed more clearly at the cell thickness below $200 \mu \mathrm{m}$, while at more than $200 \mu \mathrm{m}$, it could not be observed clearly through an 


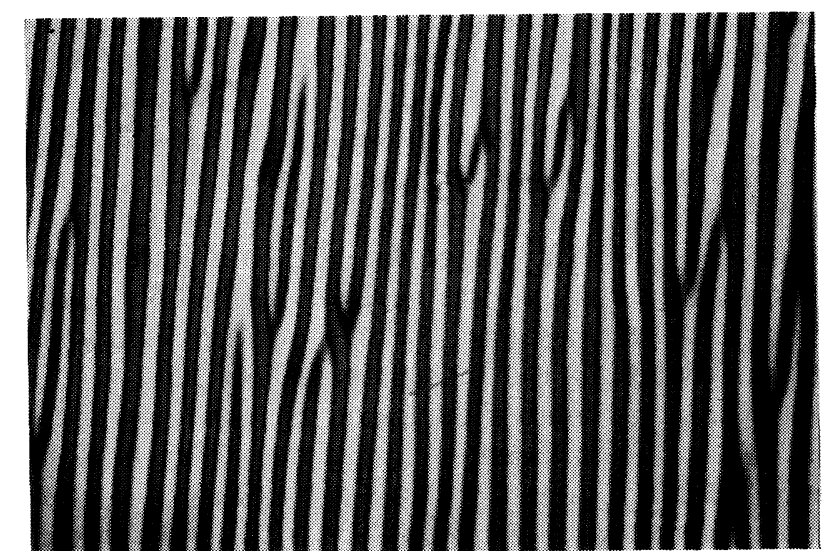

$\stackrel{100 \mu \mathrm{m}}{\longmapsto}$

(a) $10 \mathrm{~s}$

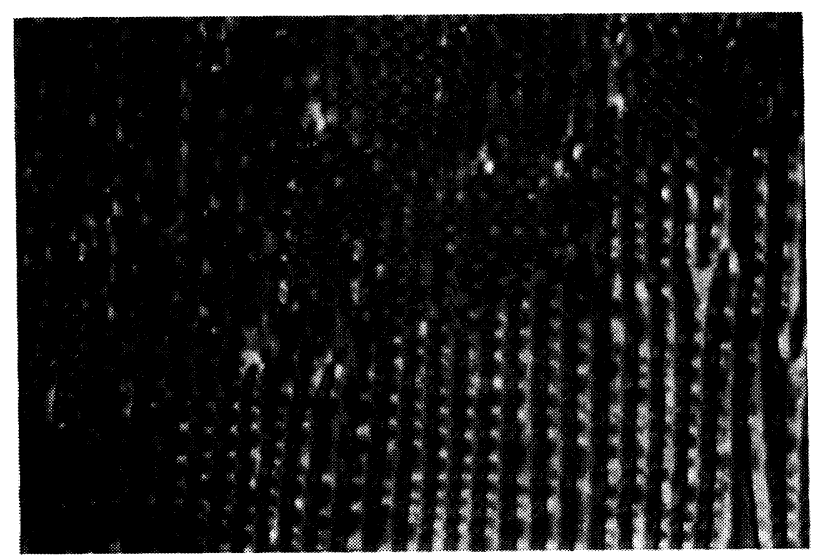

$100 \mu \mathrm{m}$

(c) $40 \mathrm{~s}$

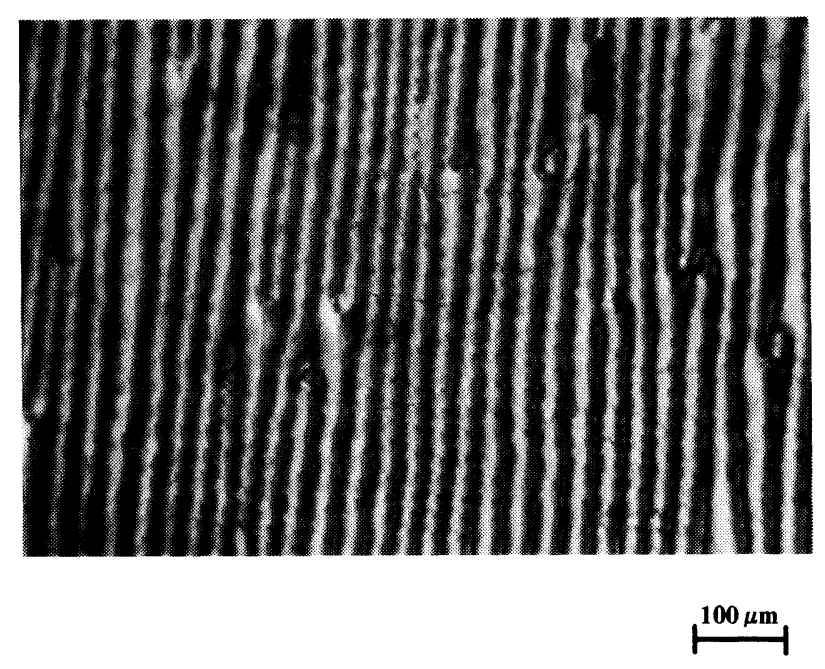

(e) $100 \mathrm{~s}$

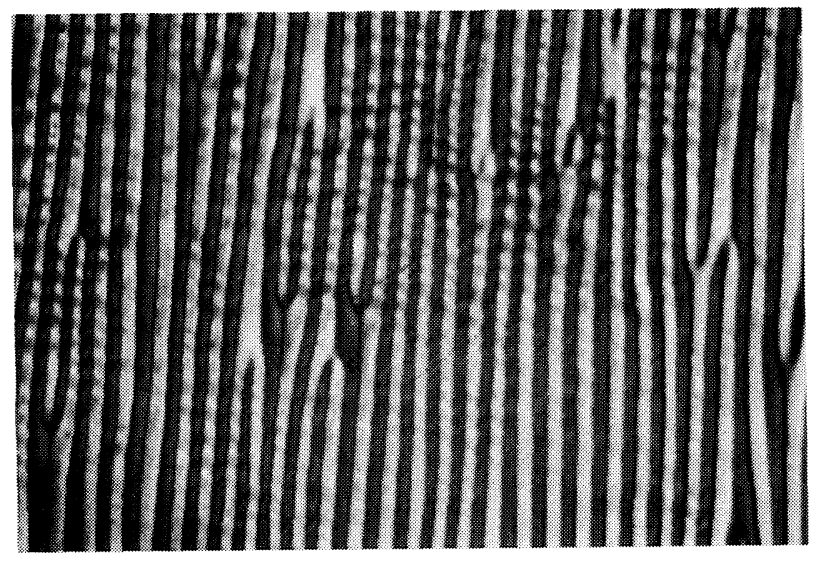

$\stackrel{100 \mu \mathrm{m}}{\square}$

(b) $20 \mathrm{~s}$.

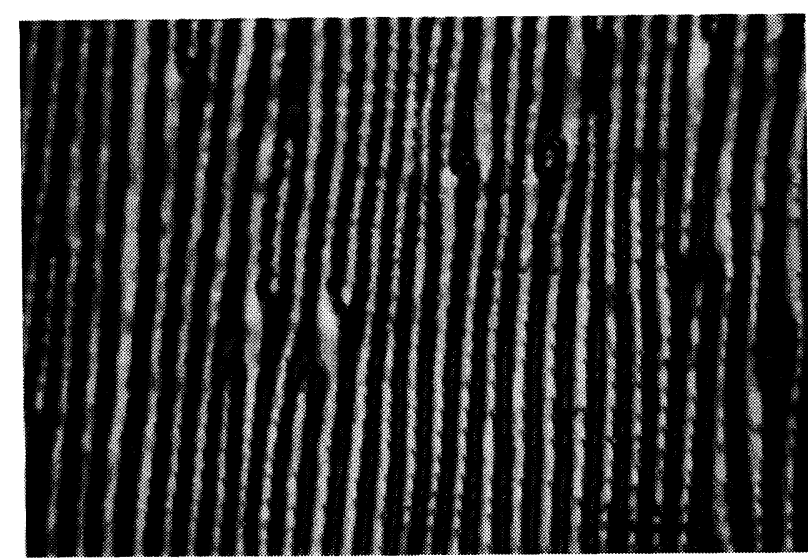

$\stackrel{100 \mu \mathrm{m}}{\longmapsto}$

(d) $80 \mathrm{~s}$

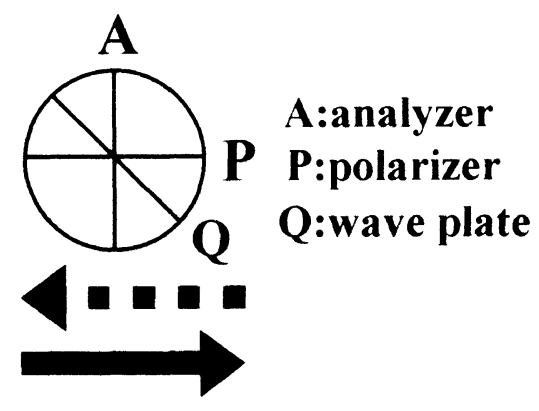

Figure 4. Development of banded texture in PBDG $\left(M_{w}=106000\right)$ liquid crystalline solution following after inversion of dc field direction. The applied field strength is $40 \mathrm{~V} \mathrm{~cm}^{-1}$ and cell thickness is $50 \mu \mathrm{m}$. Photomicrographs after field inversion, (a) $10 \mathrm{~s}$, (b) $20 \mathrm{~s}$, (c) $40 \mathrm{~s}$, (d) $80 \mathrm{~s}$, and (e) $100 \mathrm{~s}$, were taken with a wave plate inserted between crossed polars. The bar drawn under each figure indicates $100 \mu \mathrm{m}$ long. The dashed and black arrows represent the field direction before and after field inversion, respectively. 


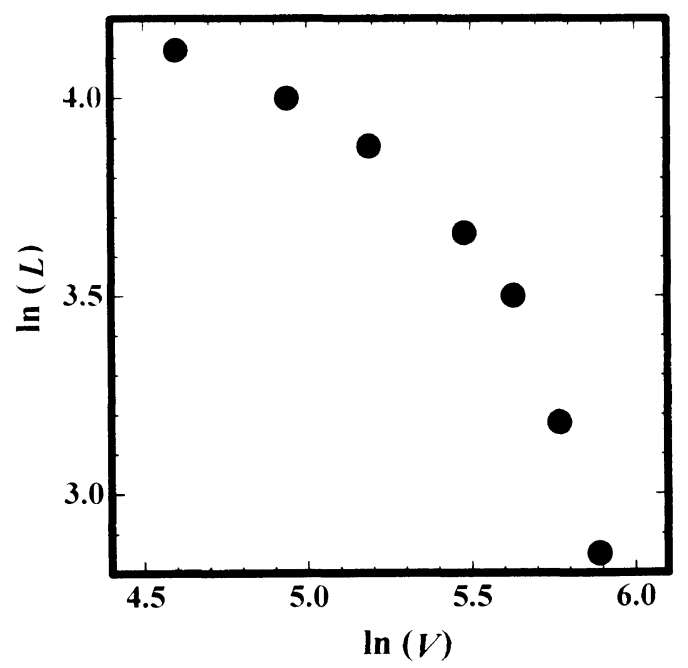

Figure 5. The relationship between the applied field strength, $V$, and band size, $L$, for PBLG $\left(M_{w}=48000\right)$ liquid crystalline solution, where cell thickness is $80 \mu \mathrm{m}$.

optical microscope because the band size, $L$, decreased with increasing cell thickness. Taking account of this, the kinetic measurements were carried out under the following conditions: at the fixed thickness of cell, 80 $\mu \mathrm{m}$, for PBLG $\left(M_{w}=48000\right)$ sample. A schematic representation of the transmittance through the whole area of the cell as scaled time after inversion of dc field direction is shown in Figure 6a, where field strength is $360 \mathrm{~V} \mathrm{~cm}^{-1}$, field direction was inverted at $t=0$ and $100 \%$ of transmittance was defined as the intensity through a vacant cell between parallel polarizers and $0 \%$ between crossed polarizers. The time between two peaks of the transmittance, $\Delta t$, as a measure of the rotational relaxation time of the directors, was determined as shown in Figure 6a. $(\Delta t)^{-1}$ is roughly proportional to $V^{2}$ for PBLG $\left(M_{w}=48000\right)$ samples in the region of higher field strength more than $240 \mathrm{~V} \mathrm{~cm}^{-1}$ as shown in Figure $6 \mathrm{~b}$. In this region, the plot is close to a straight line and so extrapolation of $(\Delta t)^{-1}$ to zero is possible. As we previously stated, the threshold, $V_{2 \mathrm{C}}$, in such a cell, $80 \mu \mathrm{m}$ of cell thickness, was $145 \mathrm{~V} \mathrm{~cm}^{-1}$. The extrapolated value is very close to this value. Recently, the response mechanism of nematic liquid crystals with applying an in-plane electric field was studied by Oh-e and Kondo. ${ }^{61}$ According to them, rise time after switching a field on, $\tau_{\text {on }}$, was inversely proportional to $\left(V^{2}-V_{\mathrm{C}}^{2}\right)$, where $V_{\mathrm{C}}$ is a threshold for IPS (in-plane switching) mode. Their results correspond to ours apparently. As already shown, the threshold for band formation is lower than $V_{2 \mathrm{C}}$ for the sample to which a field higher than the threshold $V_{2 \mathrm{C}}$ was previously applied and the precise value of the threshold for band formation could hardly be determined. The results in Figure $6 \mathrm{~b}$ suggest that the field strength tends to zero by extrapolating of $(\Delta t)^{-1}$ to zero in the range of field very close to or lower than $V_{2 \mathrm{C}}$, i.e., the apparent threshold for band formation may not exist.

We would like to stress that the local alignment and the degree of order are potentially important characters depending on the applying field strength. The timedependent distribution of the director in a domain which is recognized for a region of roughly uniform orienta-

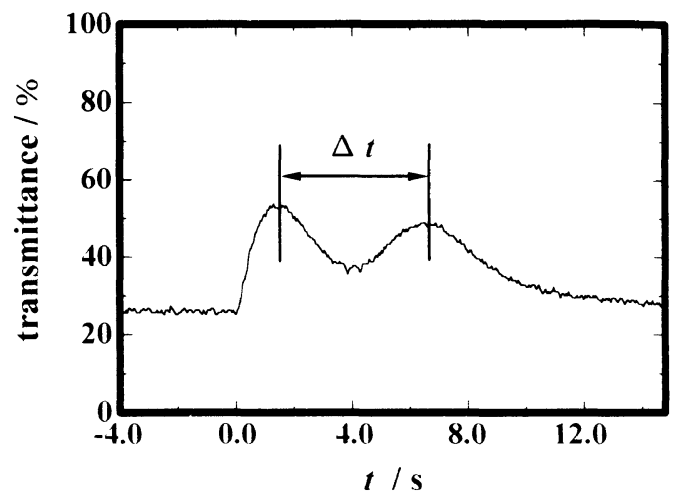

(a)

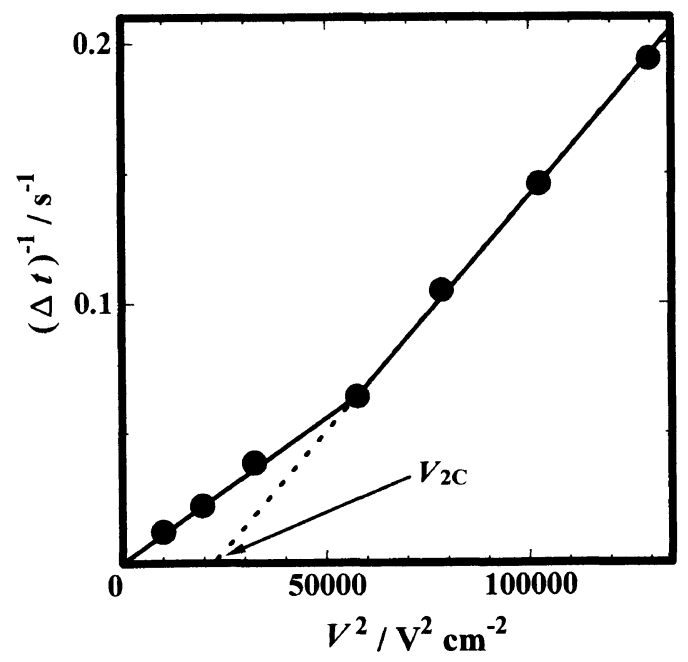

(b)

Figure 6. (a) Schematic representation of the transmittance after inversion of dc field direction for PBLG $\left(M_{w}=48000\right)$ liquid crystalline solution at $80 \mu \mathrm{m}$ of cell thickness. $\Delta t$ represents the time between two peaks as a measure of rotational relaxation time of the directors. (b) Field strength dependence of $(\Delta t)^{-1}$.

tion is also interesting. In order to examine the local movement of the director, we analyzed the variation of the light intensity transmitted through the sample sandwiched between crossed polarizers, using the images recorded on video tape. A fixed horizontal video line (200th line) of each frame at a regular interval (every 5 frames $\mathrm{s}^{-1}$ ) was picked up and the texture on the time scale was reconstructed. An example of reconstructed figures for PBLG $\left(M_{w}=48000\right)$ sample is shown in Figure $7 \mathrm{a}$, where the applied field is $180 \mathrm{~V} \mathrm{~cm}^{-1}$ and cell thickness is $80 \mu \mathrm{m}$. The vertical direction is parallel to the field direction. The axis of time, $t$, is graduated in 5 lines s ${ }^{-1}$.

A band was picked up as shown in Figure $7 \mathrm{~b}$ and the variation of intensity depending on position from line 15 to 45 and on time is illustrated in 3D figure as shown in Figure 8, where the ordinate represents the intensity with 256 gray scales. It is clearly seen that the rotational manner of director is different from line to line even within a band as shown in Figure 8 .

In order to represent the time dependence of intensity at respective positions in a domain and at domain boundaries, the intensity with gray scale on some definite pixel along the time axis in Figure $7 \mathrm{~b}$ is shown in Figure 9. As shown in Figures $9 \mathrm{a}$ and $9 \mathrm{~b}$, the intensity even at 


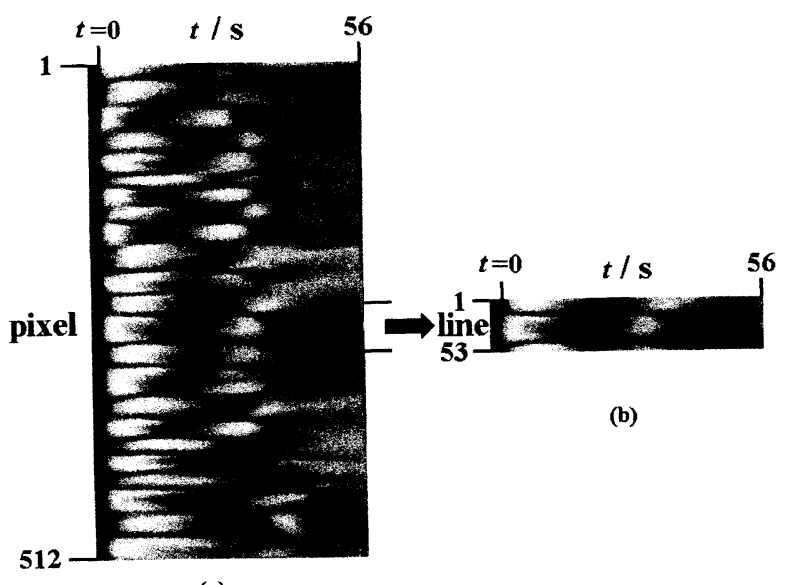

(a)

Figure 7. (a) Reconstructed figure using the image recorded on video tape for PBLG $\left(M_{w}=48000\right)$ liquid crystalline solution, where the field strength is $180 \mathrm{~V} \mathrm{~cm}^{-1}$ and cell thickness is $80 \mu \mathrm{m}$. Vertical direction is parallel to the field direction and horizontal lines are scaled with the time after inversion of the field. (b) The picked up figure from Figure 7a.

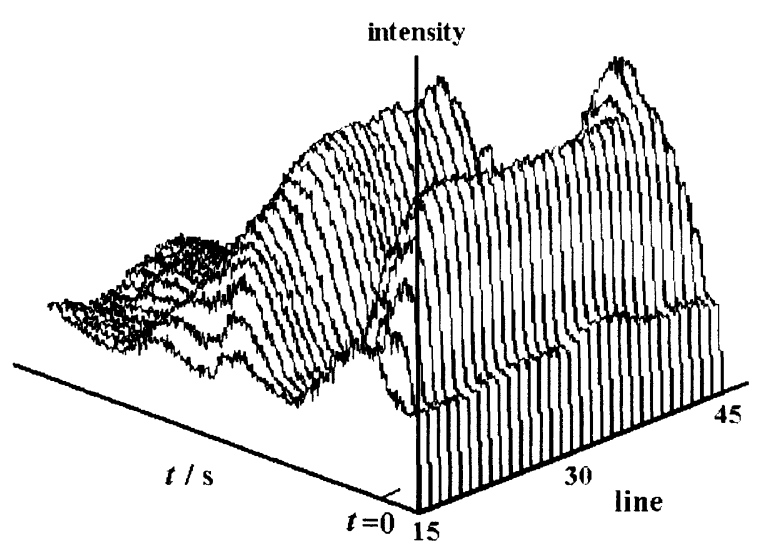

Figure 8. 3D figure represents the variation of intensity in a band shown in Figure $7 \mathrm{~b}$.

a domain boundary is not constant and hence the position of domain boundary is not immobilized. In the center part of a domain as shown in Figure 9c, the intensity changes slightly according to director rotation from place to place or from time to time with inversion.

In order to explain qualitatively this behavior, simulation for optical transmittance through two crossed polars following to rotation of the director was performed. We expect that the rotational manner of director is symmetrical from center of the cell to the upper and the lower glass plates. The distance, $h(h=d / 2$; $d$ : cell thickness), was divided into $m$ slabs parallel to the glass plate, at even intervals and the immobilized layers was assumed on both plates, where the thickness is $h_{\mathrm{s}}=(h / m) \times m_{\mathrm{s}}, m_{\mathrm{s}}$ is the number of immobilized layers. Here, the immobilized boundary layers could be often found at walls when shear stress or a magnetic field is applied for a fluid sandwiched between parallel plates. ${ }^{50}$ In this experiment, we consider that few immobilized layers exist when a rather low electric field strength is applied. Because the relation between applied field strength and $(\Delta t)^{-1}$ implies that no or only very weak anchoring may be assumed for which there is no threshold as shown in Figure 6b. Accordingly, at the beginning,
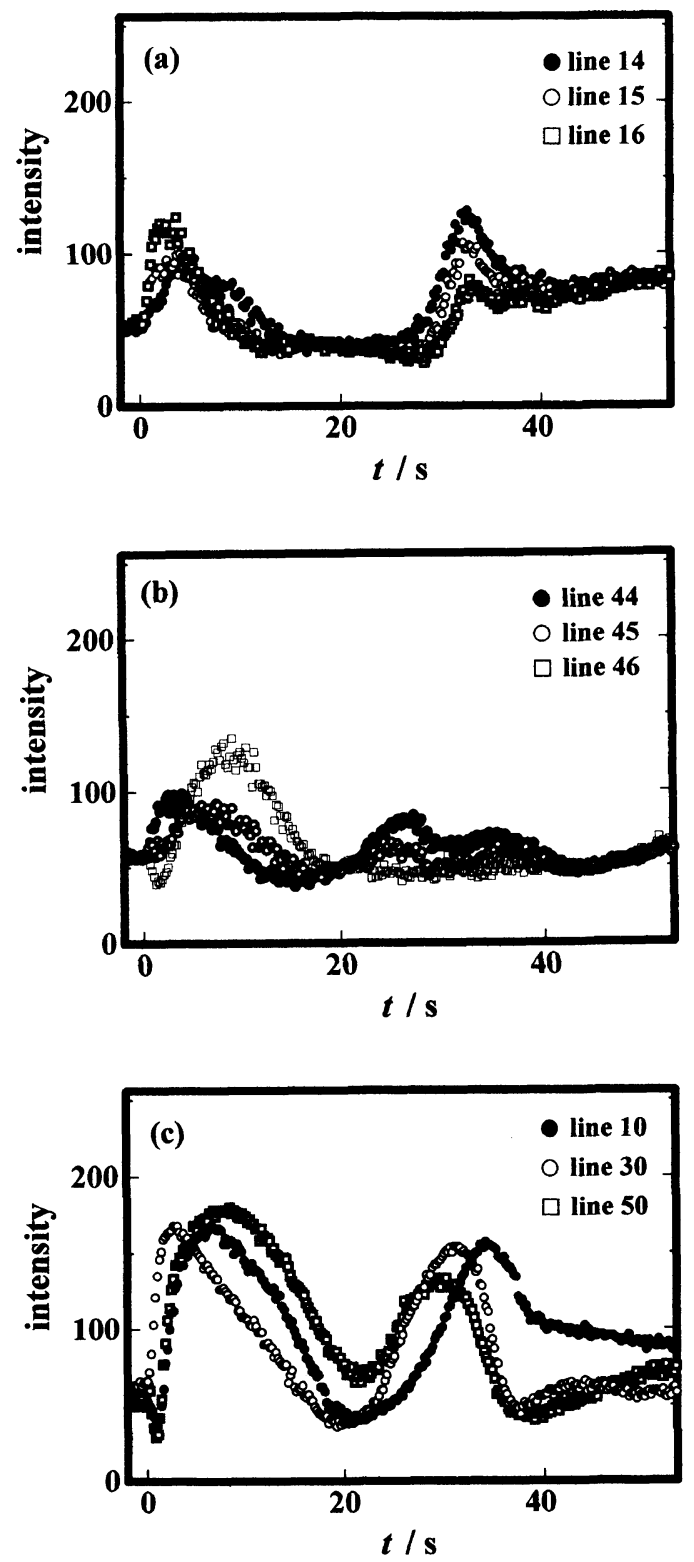

Figure 9. Time dependence of the intensity (a) at one domain boundary, (b) at the other domain boundary and (c) in the center parts of domain using Figure $7 \mathrm{~b}$.

$m_{\mathrm{s}}=0$ was assumed in this simulation. The angle between the director and the field direction, $\psi$, was assumed as

$$
\psi(t)=\pi[1-\exp (-\omega t)]
$$

where $\psi(t)$ is the angle between the director in the center slab and the direction of the electric field at any time, $t$, after inversion of the field and $\omega$ is an optional constant involved with a relaxation factor. The rotational angle of the director in a slab was assumed to change linearly along the distance, $h$, and the transmittance through all slabs from $+h$ to $-h$ was summed up. Then, the simulation for varying transmittance with rotating director after inversion of the field was performed.

The banded texture could be observed at 80,130 , and $200 \mu \mathrm{m}$ cell thickness with $360 \mathrm{~V} \mathrm{~cm}^{-1}$ of field strength. The results at each cell thickness for PBLG $\left(M_{w}=48000\right)$ samples are shown in Figure 10, where the applying dc field strength was $360 \mathrm{~V} \mathrm{~cm}^{-1}$. The simulation curves of transmittance for $h=40,65$, and 100 , assuming $\omega=0.02$, 


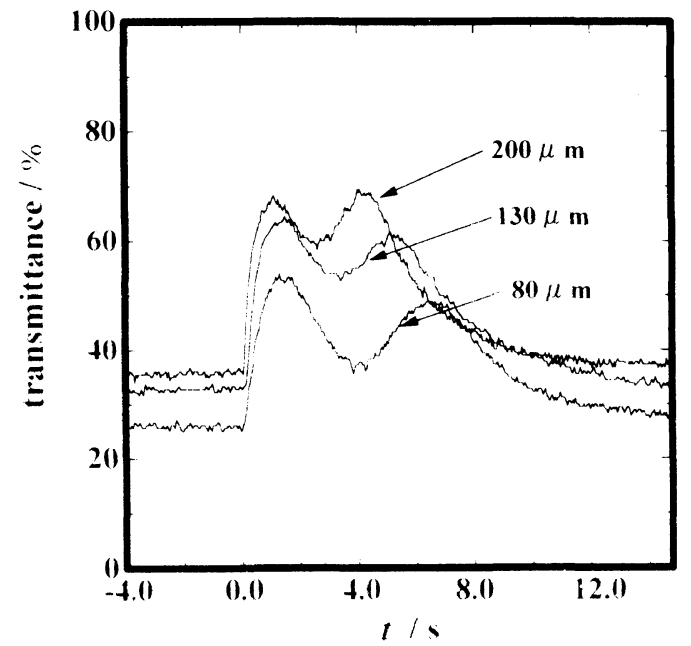

Figure 10. Time dependence of the light intensity transmitted through the whole area of the cell before and after an inversion of field direction keeping the same field strength at each cell thickness for PBLG $\left(M_{w}=48000\right)$ liquid crystalline solution, where the field strength is $360 \mathrm{~V} \mathrm{~cm}^{-1}$.

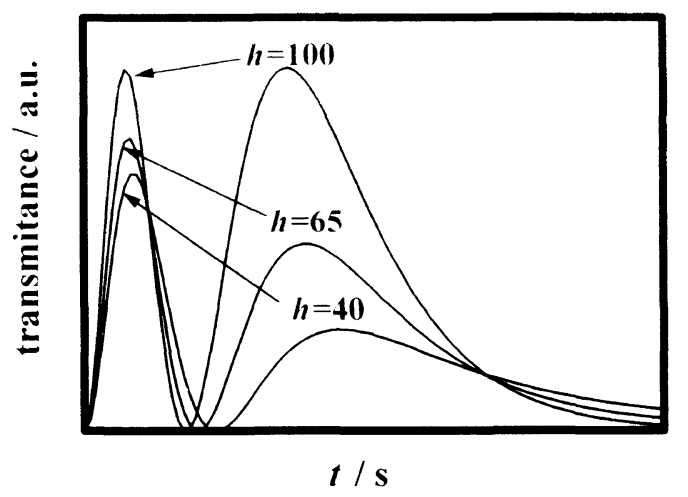

Figure 11. Simulation curves of transmittance with time passing after field inversion, where values of $h$ in this Figure correspond to real cell thicknesses, 80, 130, and $200 \mu \mathrm{m}$, respectively.

are represented in Figure 11, where the time between two peaks decrease with increasing h, i.e., cell thickness. The simulation curves correspond qualitatively to the experimental results as shown in Figure 10 except for the intensity of a trough between two peaks. That is, it is seen in Figure 10 that the transmittance at a trough between two peaks is considerably higher than that of baseline observed before field inversion. The transmittance in the Figure 10 is the sum of light intensities through the whole area of the cell. Here, the reconstructed figure and intensity with gray scale in the center part of a domain, obtained under another experimental condition, with $80 \mu \mathrm{m}$ of cell thickness and $360 \mathrm{~V} \mathrm{~cm}^{-1}$ of field strength, are shown in Figures $12 \mathrm{a}$ and $\mathrm{b}$, respectively. Obviously, the intensity at a trough between two peaks and baseline are the same level for local variation of the intensity as shown in Figure 12b and these curves are similar to the simulation curves. However, it is seen that there is some difference in the velocity of director rotation between adjacent domains as shown in Figure 12a.

\section{Rotation and Cholesteric Sense}

Rotational velocity was measured for three samples,

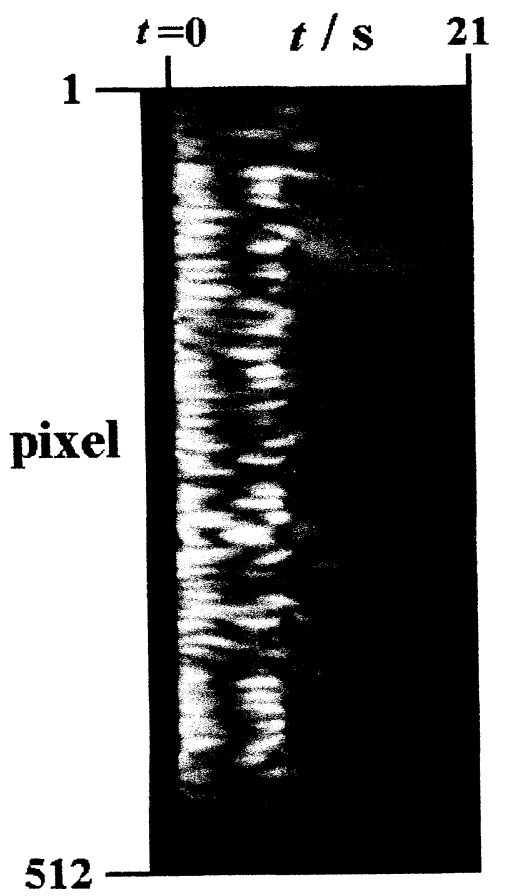

(a)

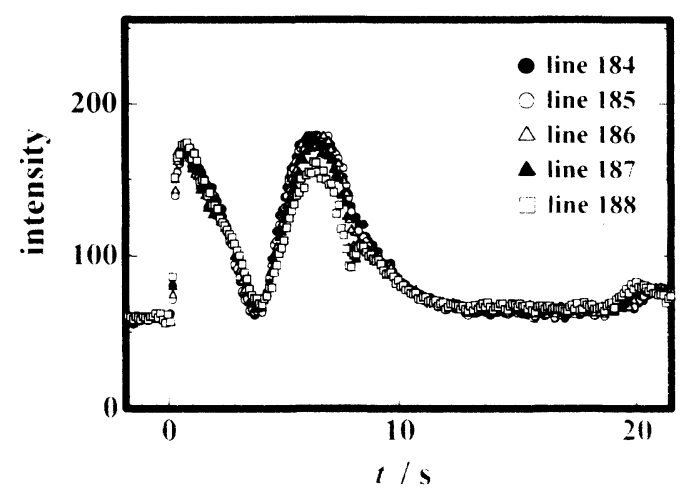

(b)

Figure 12. (a) Reconstructed figure using the image recorded on video tape for PBLG $\left(M_{w}=48000\right)$ liquid crystalline solution, where field strength is $360 \mathrm{~V} \mathrm{~cm}^{-1}$ and cell thickness is $80 \mu \mathrm{m}$. (b) Time dependence of the intensity in the vicinities of center part in a domain shown in Figure 12a.

PBLG, PBDG, and their racemic mixtures. The molecular weights of PBLG and PBDG were 96000 and 106000 , respectively. As shown in Figure 13, the rotational velocity for racemic mixture is higher than those for PBLG and PBDG, where the cell thickness used in this measurement was $50 \mu \mathrm{m}$ and the applied field strength was $40 \mathrm{~V} \mathrm{~cm}^{-1}$ as the need to use rather moderate condition arose. So far, there has been no effect of cholesteric twisting on band texture and band formation process. ${ }^{34}$ However, it is clearly seen that the effect of cholesteric twisting on the kinetics of director rotation could now be considered. Band formation could be initiated with recoiling the director. The intrinsic character of cholesterics should designate an easy direction of director rotation. Nevertheless, the experimental results show that the director rotates alternately clockwise and counterclockwise in subsequent bands in order to satisfy the requirements for electrostatic and 


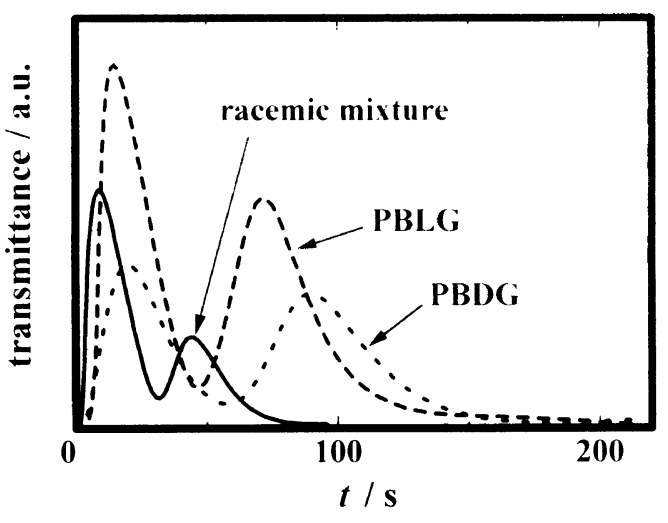

Figure 13. Transmitted intensity under crossed polars as the scaled time after inversion of dc field direction, keeping the same field strength for PBLG $\left(M_{w}=96000\right)$, PBDG $\left(M_{w}=106000\right)$, and racemic samples of PBLG and PBDG, where field strength is $40 \mathrm{~V} \mathrm{~cm}^{-1}$ and cell thickness is $50 \mu \mathrm{m}$.

elastic free-energy minima. In the kinetics of band formation and director rotation, an easy direction of director rotation must be distinguished from another direction.

\section{CONCLUSIONS}

The textural transformation induced by a dc electric field for PBLG liquid crystalline solution was observed by means of an optical polarizing microscope and the threshold values, $V_{1 \mathrm{C}}$ and $V_{2 \mathrm{C}}$, at which the texture was transformed to the other one, were determined. The threshold values were inversely proportional to the square root of cell thickness.

The banded texture perpendicular to the field direction was seen through two crossed polars soon after an inversion of field direction with keeping on the same field strength. Under our experimental conditions, the electrostatic and elastic energies can not escape through the space and then it is understood that the directors always rotate clockwise and counterclockwise in adjacent bands after inversion of a field in order to satisfy the requirement for their energy minima and/or to minimize the molecular displacement. Therefore, we observed a clear band texture and obtained quantitative data of the band size and kinetics of director rotation in a domain induced by an electric field.

Transmittance at a finite position in a domain was obtained by making use of reconstructed figure from the video-recorded texture. The results of qualitative simulation for a positional variation of optical transmittance with the local rotation of director in a domain correspond to the experimental results.

Similar banded texture and band formation except for the kinetics of director rotation were observed in the liquid crystalline solution of PBLG, PBDG, and their racemic mixture. However, the results imply that the kinetics of director rotation are affected by cholesteric twisting.

\section{REFERENCES}

1. G. Kiss and R. S. Porter, Mol. Cryst. Liq. Cryst., 60, 267 (1980). 2. M. Horio, S. Ishikawa, and K. Oda, J. Appl. Polym. Sci., Appl.
Polym. Symp., 41, 269 (1985).

3. P. Navard, J. Polym. Sci., Polym. Phys. Ed., 24, 435 (1986).

4. E. Marsano, L. Carpaneto, and A. Ciferri, Mol. Cryst. Liq. Cryst., 177, 93 (1989).

5. J. A. Odell, G. Unger, and J. L. Feijoo, J. Polym. Sci., Part B, 31, 141 (1993).

6. N. X. Yan and M. M. Labes, Macromolecules, 27, 7843 (1994).

7. N. X. Yan, M. M. Labes, S. G. Baek, and J. J. Magda, Macromolecules, 27, 2784 (1994).

8. M. G. Dobb, D. J. Johnson, and B. P. Saville, J. Polym. Sci., Polym. Symp., 58, 237 (1977a).

9. M. G. Dobb, D. J. Johnson, and B. P. Saville, J. Polym. Sci., Polym. Phys. Ed., 15, 2201 (1977b).

10. R. Qian and S. Chen, Makromol. Chem., Macromol. Symp., 53, 345 (1992).

11. P. Moldenaers and J. Mewis, J. Rheol., 30, 567 (1986).

12. J. Mewis and P. Moldenaers, Chem. Eng. Commun., 53, 33 (1987).

13. G. Marrucci, N. Grizzuti, and A. Buonaurio, Mol. Cryst. Liq. Cryst., 153, 263 (1987).

14. C. R. Fincher, Jr., Mol. Cryst. Liq. Cryst., 155, 559 (1988).

15. E. Marsano, L. Carpaneto, and A. Ciferri, Mol. Cryst. Liq. Cryst., 158B, 267 (1988).

16. E. Marsano, L. Carpaneto, A. Ciferri, and Y. Wu, Liq. Cryst., 3, 1561 (1988b).

17. E. Marsano, L. Carpaneto, and A. Ciferri, Mol. Cryst. Liq. Cryst., 177, 93 (1989).

18. B. Ernst and P. Navard, Macromolecules, 22, 1419 (1989).

19. P. L. Maffetone, N. Grizzuti, and G. Marrucci, Liq. Cryst., 4, 385 (1989).

20. R. G. Larson and D. W. Mead, J. Rheol., 33, 1251 (1989).

21. P. Moldenaers, G. Fuller, and J. Mewis, Macromolecules, 22, 960 (1989).

22. S. J. Picken, J. Aerts, H. L. Doppert, A. J. Reuvers, and M. G. Northolt, Macromolecules, 24, 1366 (1991).

23. S. J. Picken, P. Moldenaers, S. Berghmans, and J. Mewis, Macromolecules, 25, 4759 (1992).

24. J. T. Gleeson, R. G. Larson, D. W. Mead, G. Kiss, and P. E. Cladis, Liq. Cryst., 11, 341 (1992).

25. R. G. Larson and D. W. Mead, Liq. Cryst., 12, 751 (1992).

26. J. Wang and M. M. Labes, Macromolecules, 25, 5790 (1992).

27. N. Y. Yan and M. M. Labes, Macromolecules, 27, 2784 (1994).

28. S. S. Patnaik, T. J. Bunning, W. W. Adams, J. Wang, and M. M. Labes, Macromolecules, 28, 393 (1995).

29. K. Hongladarom and W. R. Burghardt, Macromolecules, 26, 785 (1993).

30. N. Grizzuti, P. Moldenaers, M. Mortier, and J. Mewis, Rheol. Acta., 32, 218 (1993).

31. R. G. Larson and D. W. Mead, Liq. Cryst., 15, 151 (1993).

32. N. J. Wagner and L. M. Walker, Macromolecules, 27, 5979 (1994).

33. C. Viney and W. S. Putnam, Polymer, 36, 1731 (1995).

34. J. Vermant, P. Moldenaers, J. Mewis, and S. J. Picken, J. Rheol., 38, 1571 (1994).

35. A. M. Donald, C. Viney, and A. H. Windle, Polymer, 24, 155 (1983).

36. A. M. Donald and A. H. Windle, J. Mater. Sci., 18, 1143 (1983).

37. C. Viney, A. M. Donald, and A. 'H. Windle, J. Mater. Sci., 18, 1136 (1983).

38. D. J. Graziano and M. R. Mackley, Mol. Cryst. Liq. Cryst., 106, 73 (1984).

39. A. E. Zachariades, P. Navard, and J. A. Logan, Mol. Cryst. Liq. Cryst., 110, 93 (1984).

40. Y. Takeuchi, Y. Shuto, and F. Yamamoto, Polymer, 29, 605 (1988).

41. P. K. Chan and A. D. Rey, Liq. Cryst., 12, 1025 (1992).

42. P. K. Chan and A. D. Rey, Liq. Cryst., 13, 775 (1993).

43. E. Guyon, R. Meyer, and J. Salan, Mol. Cryst. Liq. Cryst., 54, 261 (1979).

44. R. G. Larson and H. C. Ottinger, Macromolecules, 24, 6270 (1991).

45. R. G. Larson and M. Doi, J. Rheol., 35, 539 (1991).

46. G. Marrucci, Pure Appl. Chem., 57, 1545 (1985).

47. S. A. Patlazhan, J. B. Riti, and P. Navard, Macromolecules, 29, 2029 (1996).

48. W. H. Daly and D. Poché, Tetrahedron Lett., 29, 5859 (1988).

49. K. Monzen, K. Hiraoka, Y. Uematsu, and M. Date, Mol. Cryst. Liq. Cryst., 299, 175 (1997). 
Banded Texture in a dc Field

50. O. Parodi, Solid State Commun., 11, 1503 (1972).

51. M. Goscianski, L. Leger, and A. Mircea-Roussel, J. Physique Lett., 36, L-313 (1975).

52. D. W. Allender, B. J. Frisken, and P. Palffy-Muhoray, Liq. Cryst., 5, 735 (1989).

53. B. J. Frisken and P. Palffy-Muhoray, Phys. Rev. A., 40, 6099 (1989).

54. B. J. Frisken and P. Palffy-Muhoray, Phys. Rev. A., 39, 1513 (1989).

55. A. M. Donald, C. Viney, and A. P. Ritter, Liq. Cryst., 1, 287
(1986).

56. G. Marrucci, N. Grizzuti, and A. Buonaurio, Mol. Cryst. Liq. Cryst., 153, 263 (1987).

57. B. Ernst, P. Navard, T. Hashimoto, and T. Takebe, Macromolecules, 23, 1370 (1990).

58. E. Peuvrel and P. Navard, Liq. Cryst., 7, 95 (1990).

59. K. Monzen, D. Fujita, K. Hiraoka, Y. Uematsu, and M. Date, Mol. Cryst. Liq. Cryst., 303, 51 (1997)

60. M. Oh-e and K. Kondo, Appl. Phys. Lett., 69, 623 (1996). 